\title{
The decreased susceptibility of metastatic melanoma cells to killing involves an alteration of CTL reactivity
}

\author{
AHMED GASSARA $^{1 *}$, YOSRA MESSAI $^{1 *}$, CATHERINE GAUDIN $^{1}$, SORAYA ABOUZAHR $^{1}$, \\ ADBELALI JALIL ${ }^{1}$, MARYAM DIARRA-MEHRPOUR ${ }^{1}$, FLORENCE FAURE ${ }^{3}$, CATHERINE RICHON ${ }^{1}$, \\ MARIE-FRANÇOISE AVRIL ${ }^{2}$, JOS EVEN $^{1 * *}$ and SALEM CHOUAIB ${ }^{1 * *}$ \\ ${ }^{1}$ Unit 753 INSERM, ${ }^{2}$ Department of Medicine, Institut Gustave Roussy, 39 rue Camille Desmoulins, \\ 94805 Villejuif; ${ }^{3}$ Unit 653 INSERM, Institut Curie, 26 rue d'Ulm, 75248 Paris, France
}

Received December 19, 2005; Accepted January 30, 2006

\begin{abstract}
Metastases are known to be more resistant to therapy than matching primary tumors, in particular they are less prone to apoptosis. In this study we investigated the functional interaction of a CTL clone (LT12) specific for a melanoma TA with the primary tumor (T1) versus its metastatic counterpart (G1). The CTL clone (LT12) was shown to lyse the primary $\mathrm{T} 1$ cells more efficiently in a classical cytotoxicity test. This differential susceptibility was not associated with MHC class I down-regulation and conjugate formation but correlated with a differential increase in $\mathrm{Ca}^{++}$flux in the LT12 CTL when stimulated with the primary versus the metastatic tumor cells. Since LT12 uses perforin/granzyme B to kill its autologous target we analysed perforin and granzyme B mRNA expression in the CTL in the presence of either primary and metastatic melanoma cells. Quantitative PCR analysis showed an increased expression of granzyme B and perforin mRNA levels in LT12 when cocultured in the presence of the primary tumor. However, a similar level of (cytotoxic molecule) degranulation as revealed by CD107 expression was observed when LT12 was stimulated with T1 or G1 cells. These data suggest that the differential susceptibility of primary and metastatic melanoma cells involves at least in part their distinct potential to induce autologous CTL reactivity and the subsequent triggering of granzyme B and perforin in these cells.
\end{abstract}

Correspondence to: Dr Salem Chouaib, INSERM U753, Institut Gustave Roussy, 39 rue Camille Desmoulins, 94805 Villejuif Cedex, France

E-mail: chouaib@igr.fr

${ }^{*}$ Contributed equally

${ }^{* *}$ Co-senior authors

Abbreviations: mAb, monoclonal antibody; CTL, cytotoxic T lymphocyte; MICA, MHC class I-related chain A; MICB, MHC class I-related chain B

Key words: CTL, primary melanoma, metastatic melanoma

\section{Introduction}

Melanoma is the tumor with the fastest growing incidence among Caucasians. While early detection and surgery of primary melanoma improves the outcome, metastatic melanoma is still largely refractory to therapy (1). As melanoma progresses to metastatic disease, powerful mechanisms of tumor evasion from immune recognition are activated thus tilting the balance between immunity and escape in favor of tumor resistance to host defense. Thanks to recent progress in tumor immunology, immunotherapy trials have been carried out; however, very little success has been achieved so far (2). At the present time, the molecular pathogenesis of melanoma is still largely unknown and both classical and biological tumor therapy approaches to cure metastatic melanoma are largely inefficient. In this regard, expression profiling has identified clusters and identified genes of interest that could be targeted in future therapies (3). Furthermore, response to IL-2 therapy defines clusters of response, as with other sets of data, no correlation with clinical outcome was established for melanoma patients $(3,4)$.

The most effective immunologically mediated host strategy is the deployment of cytotoxic T lymphocytes that can directly lyse targets and have the capacity to secrete effector cytokines such as interferon $\gamma$ and tumor necrosis factor $\alpha(5)$. In the $\mathrm{T}$ cell-mediated cytotoxicity process, two major pathways are engaged following TCR recognition of $\mathrm{Ag} / \mathrm{MHC}$ complexes expressed on target cells. The first one is a secretory pathway involving receptor-triggered exocytosis of performed secretory granules containing granzymes and perforin $(6,7)$. The second is based on receptor-induced surface expression of death receptor ligands on effector cells, which cross-links the corresponding receptors (Fas, TRAIL receptors, TNFR I-p55) on target cells (8). In mice and humans, granzymes A and B are the most abundant granzymes and have received the most attention, in particular granzyme B. Several lines of evidence indicate that tumor cells proliferate under adverse host conditions during tumor progression, using several strategies to adapt their survival by blocking the action of key regulators of the immune response and circumventing anti-tumor defenses. Despite the expression of tumor-associated antigens, tumor eradication by the immune system is often inefficient (9). In 
this regard, it has been reported that metastatic cancer cells exhibit a higher resistance to apoptosis as compared to their poorly-metastatic counterparts, which indicates that metastatic cancer cells may have more dysregulations in apoptosis pathways (10-12). However, there are only few experimental data linking tumor susceptibility to CTL-induced cell death and metastasis (13-16). It is well established that the outcome of the antitumor response is the result of cross-talk between effector cells and the tumor target submitted to the influence of the tumor microenvironment. Therefore, the present study was undertaken to investigate the primary versus metastatic tumor susceptibility to CTL-mediated killing and their reciprocal functional interaction with these effectors. For this purpose, we used a previously described system comprising a CTL clone, LT12, and the autologous primary T1 and metastatic G1 melanoma cell lines (17). We show that besides the differential susceptibility to lysis, primary and metastatic targets induce a differential reactivity of the autologous CTL clone.

\section{Materials and methods}

Cells. Both primary tumor and lymph node metastases were obtained in 1995 from patient Fon, a 77-year-old male with a primary facial melanoma lesion. Tumor samples were mechanically dissociated and aliquots of the resulting single cell suspension maintained in culture for both tumor-infiltrating lymphocyte (TIL) expansion and tumor cell line generation. The LT 12 CTL clone was isolated from autologous TIL as described previously (12). T1 Fon and G1 Fon melanoma cell lines were derived from the primary lesion (T1) and from an invaded lymph node $(\mathrm{G} 1)$.

Cytolytic activity assay. Standard $4-\mathrm{h}{ }^{51} \mathrm{Cr}$ release assays were used to assess antigen-specific target cell lysis. Target cells $\left(10^{6}\right)$ were labelled with $100 \mu \mathrm{Ci} \mathrm{Na}{ }_{2}^{51} \mathrm{CrO}_{4}$ (Perkin-Elmer Life Sciences, St. Laurent, Quebec) for $1 \mathrm{~h}$ at $37^{\circ} \mathrm{C}$, washed three times with RPMI media and then diluted to $2 \times 10^{4}$ cells $/ \mathrm{ml}$ in complete media. In triplicate, $100 \mu \mathrm{l}$ of target cells were seeded into 96-well V-bottom Libro/Titertek tissue culture plates (ICN Biomedicals, Aurora, OH) with $100 \mu 1$ effector cells (to give effector:target ratios of 30:1, 10:1, 5:1 and 1:1) and cultures were then incubated for $4 \mathrm{~h}$ at $37^{\circ} \mathrm{C}$. Released ${ }^{51} \mathrm{Cr}$ from supernatants was measured on a Wallac $1470 \gamma$-counter (Wallac Oy, Turku, Finland). Results were expressed in lytic units (LU). LU were calculated as described by Pross et al (18). One LU was defined as the number of effector cells required for $30 \%$ lysis of $3 \times 10^{3}$ target cells and the number of LU present in $10^{6}$ effector cells was calculated using a computer program. Inhibition of the granzyme mediated cytotoxic pathway was performed by preincubation of effector cells for $2 \mathrm{~h}$ with $100 \mathrm{nM}$ concanamycin A (CMA) (Sigma, France). Target cells were then added to the wells with CMA-treated effector cells (concentration of CMA during lysis: $50 \mathrm{nM}$ ).

Antibodies. T1 and G1 cells were stained with a panel of monoclonal antibodies (mAb) which included anti-HLA class I (19), anti-MICA (M673, Amgen), and anti-MICB (M364, Amgen). mAb were used to stain cells in an indirect immunofluorescence. Following incubation, the cells were washed and analysed in a FACScalibur ${ }^{\mathrm{TM}}$ (BD Bioscience, Le Pont de Claix, France).

Measurement of TNFß production by CTLs. LT12 cells were incubated with T1 or G1 tumor cells at a lymphocyte:tumor ratio of 1:6. The supernatents were collected after $12 \mathrm{~h}$, and the TNFß content was detected by measuring the cytotoxicity of the culture medium on the TNF-sensitive WEHI-164c13 cells, with a 3-(4,5-dimethylthiazol-2-yl)-2,5-diphenyl tetrazolium bromide colorimetric assay (20).

Analysis of raft distribution by confocal microscopy. As previously described (21), T cells were incubated with $8 \mu \mathrm{g} / \mathrm{ml}$ of cholera toxine Alexa Fluor conjugate (Molecular Probes) for $35 \mathrm{~min}$ in ice-cold PBS. After washing, CT-loaded T cells were dropped onto target cells previously attached to a poly-L-lysine-coated slide. Confocal microscopy LSM510 (Zeiss, Hallbergnoos, Germany) was performed at 5, 30, 60 and 120 min of coculture.

Videomicroscopy and intracellular calcium concentration assessment. T cells were labelled with $3 \mu \mathrm{M}$ of Fluo4-AM (Molecular Probes, OR) for $15 \mathrm{~min}$ at $37^{\circ} \mathrm{C}$, washed twice and incubated in RPMI-1640 + 10\% FCS for 30 min before coculture with target cells on a glass slide. Video microscopy was performed for $1 \mathrm{~h}$, in HEPES-buffered medium in a temperature-controlled chamber. Pictures were acquired every $10 \mathrm{sec}$. An increase in fluorescence intensity of at least $50 \%$ above the background level was considered significant for calcium influx.

CD107a lysosome-associated membrane protein (LAMP-1) expression assay. Degranulation of intracellular vesicles by lymphocytes can be measured using CD107a, as described recently for $\mathrm{CD}^{+} \mathrm{T}$ cells (22). Tumoral cells and $\mathrm{CD} 8^{+}$effector cells, were mixed in a 1:2 target:effector ratio in the presence of $1 \mu \mathrm{g} / \mathrm{ml} \mathrm{CD107a-PeCy5} \mathrm{Ab} \mathrm{(BD} \mathrm{Biosciences)} \mathrm{for} \mathrm{30,} \mathrm{60,}$ 90 or $180 \mathrm{~min}$ at $37^{\circ} \mathrm{C}$. Samples were then surface-stained using CD3-FITC (BD Biosciences) for $30 \mathrm{~min}$, washed and fixed with $3.7 \%$ paraformaldehyde (Sigma-Aldrich). Flow cytometry analysis for cell surface CD107a expression was performed on gated lymphocytes.

Real-time quantitative reverse transcription-PCR analysis. For analysis of granzyme B and perforin induction, LT12 was conjugated with $\mathrm{T} 1$ or G1 cells at a 2:1 ratio for 2 and $3 \mathrm{~h}$. RNA extraction was performed with TRIzol and reverse transcribed. PCR primers and probes for the granzyme B and perforin genes were designed by Applied Biosystems and used according to the manufacturer's recommendations. The amount of sample RNA was normalized by the amplification of an endogenous control (18S). The relative quantification of the transcripts was derived using the standard curve method (Applied Biosystems User Bulletin 2, ABI PRISM 7700 Sequence Detection system).

\section{Results}

Differential susceptibility of primary $T 1$ and metastatic G1 melanoma cells to killing by autologous CTL clone. We first 


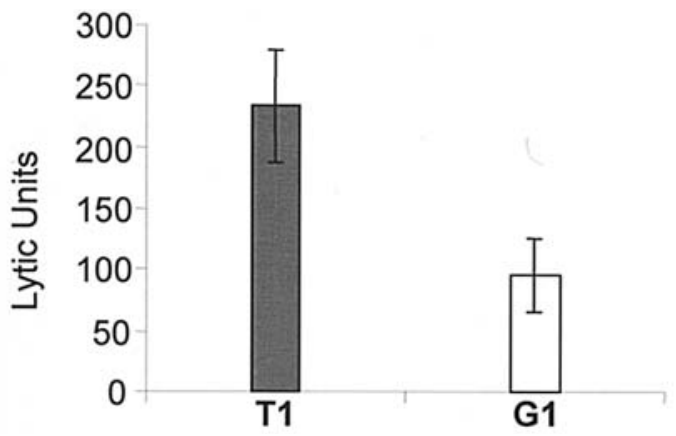

Figure 1. LT12 mediated killing of primary (T1) and metastatic (G1) melanoma cells. Chromium $\left({ }^{51} \mathrm{Cr}\right)$ labelled melanoma cells were incubated at $37^{\circ} \mathrm{C}$ with LT12 CTL cells at different ratios. After $4 \mathrm{~h}$, the supernatants were collected and analysed in a $\gamma$-counter. Lysis was expressed as lytic units (LU-30). One lytic unit is defined as the number of LT12 cells required for $30 \%$ lysis of $3 \times 10^{3}$ target cells and the number of LU present in $10^{6}$ effector cells is presented.

investigated the susceptibility of the primary melanoma cell line, T1, and its metastatic derivative, G1, to the cytotoxicity induced by the autologous CTL clone, LT12, using the standard chromium release assay. As shown in Fig. 1, while primitive melanoma cells were significantly killed (234 LU) by the CTL clone, a significant decrease in susceptibility to lysis (96 LU) was observed when G1 metastatic melanoma cells were used as targets.

The differential susceptibility of primary and metastatic targets is not due to down-regulation of MHC class I molecule expression. The loss of MHC class I by metastatic cells has been previously reported $(23,24)$. In order to examine whether a relationship exists between expression of MHC class I and decreased susceptibility to CTL killing, we investigated the expression of MHC class I, MICA and MICB on T1 and G1 cells. Using flow cytometry analysis, we demonstrated a similar surface expression of MHC class I molecules on T1 and G1 cells as revealed by cell staining with W6/32 antibody (Fig. 2A). Because LT12 expresses a high level of NKG2D (data not shown), we also examined its ligand expression by primary and metastatic tumor cells. The data depicted in this figure show that the expression of MICA and MICB by T1 and G1 cells was similarly low and suggest that the observed resistance of metastatic cells to CTL lysis is independent of class I MHC expression.

Furthermore, TNF secretion by LT12 was evaluated in order to examine target recognition by the LT12 CTL clone. As depicted in Fig. 2B, a similar amount of TNF was secreted by the CTL clone stimulated with T1 or G1 cells, ruling out impaired cellular antigen processing and/or presentation.

Conjugate formation between the CTL and primary or metastatic tumor cells. Accumulated actin at the contact between $\mathrm{T}$ and antigen presenting cells (APCs) was found to stabilize a continuous contact that allows the clustering of TCRs and costimulatory signaling molecules into lipid rafts (25). As shown in Fig. 3, using confocal microscopy, we observed rapid conjugate formation (at $5 \mathrm{~min}$ ) between the LT12 CTL clone and T1 or G1 tumor cells. This figure shows a tight polarization of the fluorescence at contact site with similar lipid
A

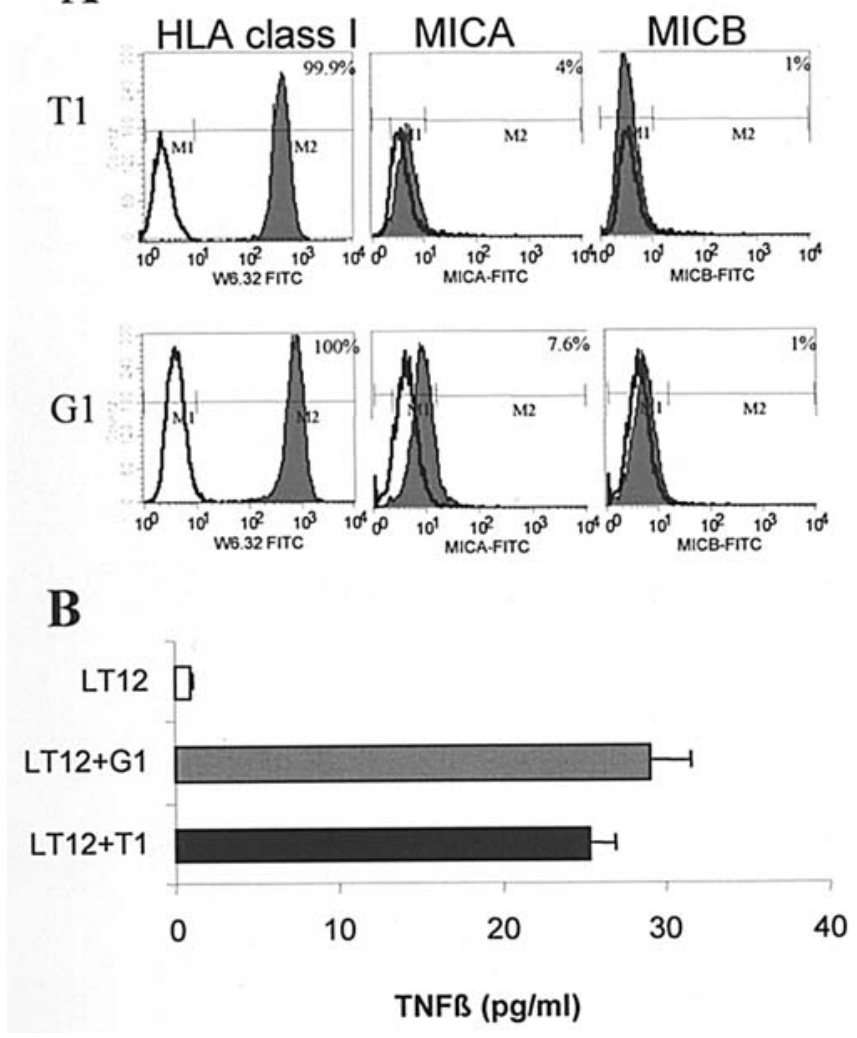

Figure 2. (A) Cell surface expression of MHC class I, MICA and MICB on target cells. T1 and G1 were stained with FITC-conjugated anti-MHC class I, anti-MICA and anti-MICB. Black-shaded histograms are for positive cells and white-shaded histogram for mouse isotype control ( $\mathrm{IgG})$. Values indicate the percentage of positive cells. (B) TNFß production by cytotoxic $\mathrm{T}$ lymphocytes in response to the primary and the metastatic cells. After $12 \mathrm{~h}$ of coculture of CTL and tumor cells, the supernatants were analysed by an MTT test in triplicate.

raft coalescence in the presence of both primary and metastatic cells. This suggests that the decreased susceptibility of metastatic melanoma cells to killing is not the likely consequence of an alteration of aggregation of lipid rafts and thus not attributable to a defect in cellular interaction between effector CTL and target cells.

Differential calcium flux in autologous CTL in the presence of primitive and metastatic melanoma. Having shown the differential susceptibility of primary versus metastatic cells to CTL-mediated lysis, we then analysed the $\mathrm{Ca}^{2+}$ response in LT12 CTL as a measure for early T cell recognition and reactivation events. LT12 CTL were labelled with Fluo4-AM before incubation in the presence of either $\mathrm{T} 1$ or $\mathrm{G} 1$ melanoma cell lines. The $\mathrm{Ca}^{2+}$ response was assessed using video microscopy. As shown in Fig. 4, increased calcium influx was observed in LT12 CTL when cocultured with the primary T1 cells. However, when G1 metastatic cells were used for stimulation, only a marginal increase in $\mathrm{Ca}^{+}$flux was observed in the LT12 CTL clone.

LT12 uses the perforin/granzyme pathway to kill T1 and G1 targets. T1 and G1 cells were used as targets of CTL in the presence or absence of CMA (to chelate $\mathrm{Ca}^{2+}$ ). As shown in Fig. 5A, an inhibition of exocytosis mediated pathway by 


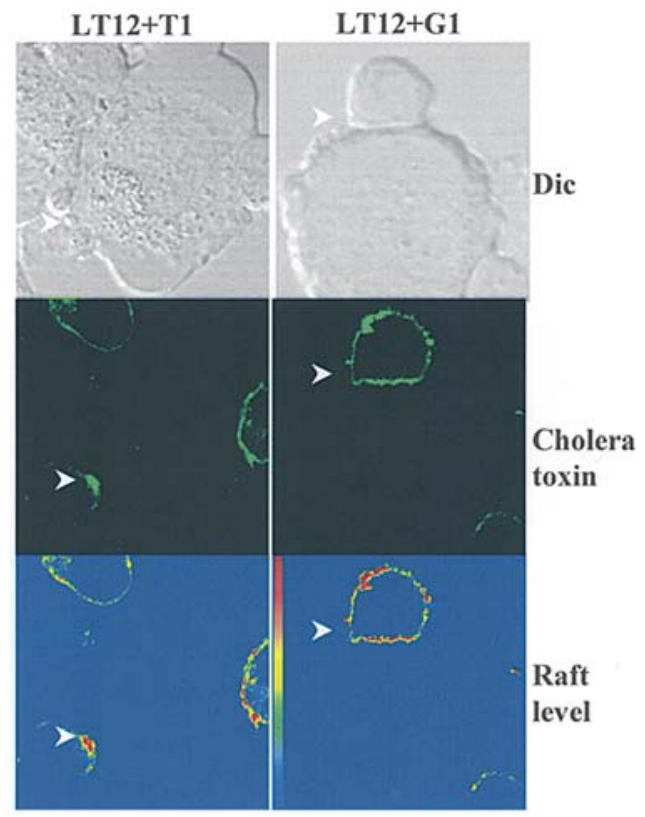

Figure 3. Immunological synapse formation between LT12 CTL and the primary or metastatic cells. After 5, 30,60 and $120 \mathrm{~min}$ of coculture at $37^{\circ} \mathrm{C}$, the cells were fixed with $4 \%$ paraformaldehyde and mounted on Vectashield. Conjugates were examined using confocal microscopy. Synapse formation at $5 \mathrm{~min}$ of coculture is shown. Data are from one representative experiment of three. concamycin A resulted in the abrogation of LT12 CTL clone cytotoxicity, indicating that the observed cytotoxicity towards both $\mathrm{T} 1$ and $\mathrm{G} 1$ cells is mediated by the perforin/granzymes pathway. Further, we examined the degranulation ability of CTLs in the presence of primary or metastatic cells. As illustrated in Fig. 5B, the percentage of degranulation was similar at different times following LT12 conjugation with $\mathrm{T} 1$ targets or $\mathrm{G} 1$ targets.

Down-regulation of perforin, granzyme $B$ mRNA expression in LT12 CTL stimulated by metastatic tumor cells. We have shown that the LT12 CTL clone mainly used the secretory pathway to lyse T1 and G1 targets. To investigate the stimulatory potential of T1 primary and G1 metastatic tumor cells, LT12 CTL clone was incubated in the presence of either T1 or G1 cells. Quantitative PCR analysis demonstrated that stimulation of the CTL clone by T1 cells resulted in significant granzyme B transcription while no change in this gene transcription was observed when G1 cells were used as stimulatory cells (Fig. 6). Fig. 6 also shows that, in LT12 cells stimulated either with $\mathrm{T} 1$ or G1 cells, perforin gene transcription was very low when LT12 was stimulated with G1 cells. Such transcription even decreased following stimulation with both cell types, confirming that granzyme B and perforin are not under the same transcriptional control in activated CTLs.
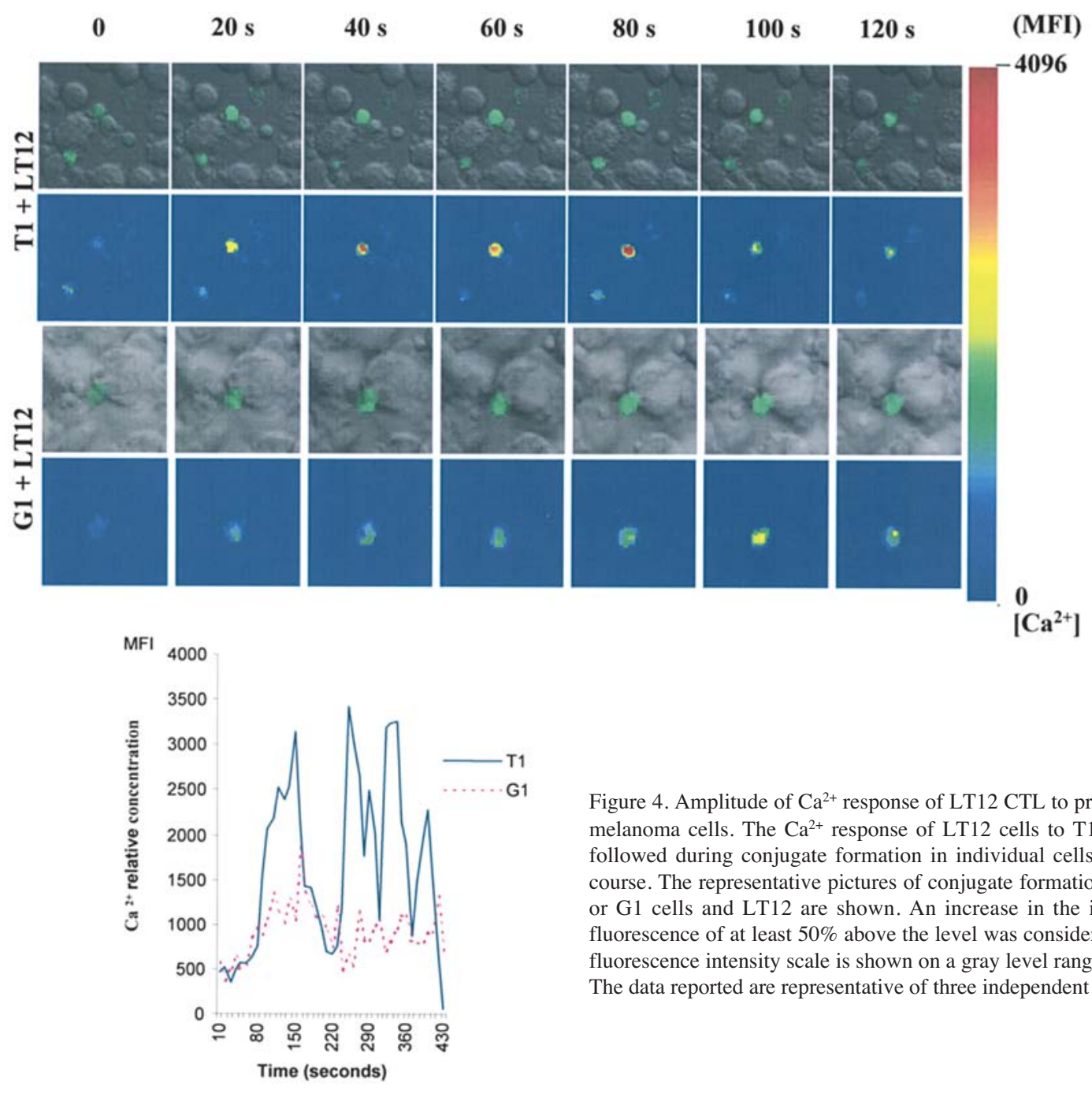

Figure 4. Amplitude of $\mathrm{Ca}^{2+}$ response of LT12 CTL to primary or metastatic melanoma cells. The $\mathrm{Ca}^{2+}$ response of LT12 cells to T1 and G1 cells was followed during conjugate formation in individual cells during a 1 -h time course. The representative pictures of conjugate formation between T1 cells or G1 cells and LT12 are shown. An increase in the intensity of Fluo-4 fluorescence of at least $50 \%$ above the level was considered significant. The fluorescence intensity scale is shown on a gray level ranging from 0 to 4096 . The data reported are representative of three independent experiments. 

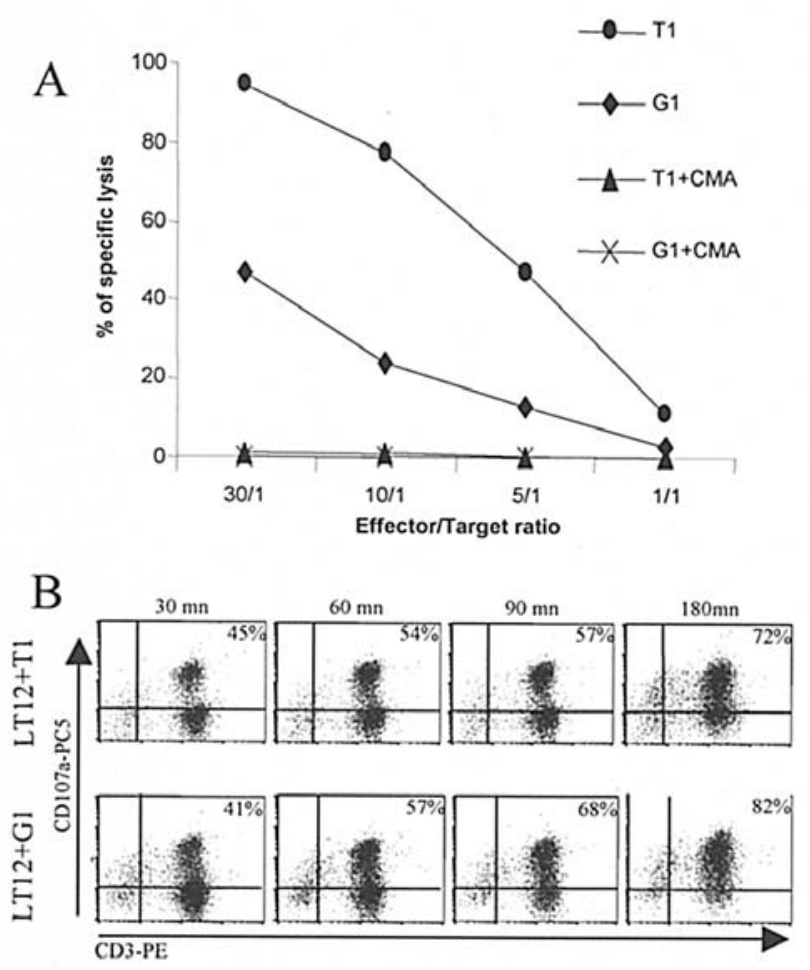

Figure 5. (A) CMA blocks LT12 CTL killing of T1 and G1 cells. Cytotoxicity was determined using a $4-\mathrm{h}{ }^{51} \mathrm{Cr}$ release assay in the presence or absence of $\mathrm{Ca}^{2+}$ chelator CMA. (B) CD107a expression assay. LT12 CTLs were stained with PC5-conjugated anti-CD107a and PE-conjugated antiCD3 after 30, 60, 90 and 180 min of coculture with T1 and G1 cells. Values indicate percentage of positive cells.
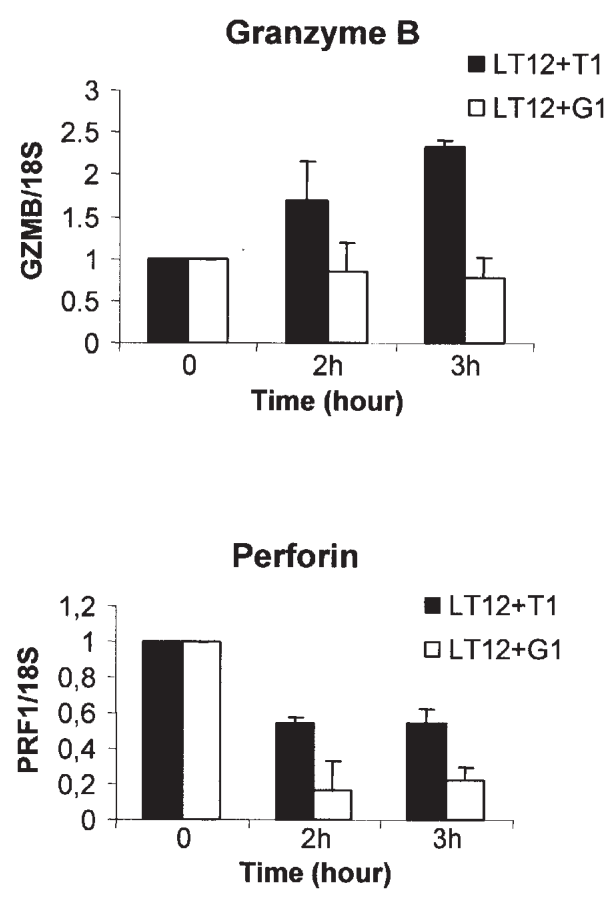

Figure 6. mRNA expression of perforin, granzyme B in LT12 CTL. LT12 was cocultured with T1 or G1 cells for 120 and $180 \mathrm{~min}$. At the end of coculture, cells were collected and the total RNA was extracted and followed by quantitative PCR analysis for granzyme B and perforin gene expression. The amount of sample RNA was normalized by the amplification of an endogenous control (18S). The mixture of LT12 with T1 or G1 cells without incubation (represented by a time of $0 \mathrm{~min}$ ) was used for calibration. The data reported are representative of two independent experiments.

\section{Discussion}

In human melanoma, early detection and excision of primary tumors has improved prognosis but treatment of metastases is still very inefficient with 5-year survival rates of $6 \%$ (26). Although, metastatic cells were previously found to be more resistant to apoptosis induced by cytotoxic treatments (12), little is known about the relative susceptibility of metastatic tumor cells to CTL-mediated lysis. Thanks to important progress made over the past ten years, biotherapy and, in particular, targeted immunotherapy protocols could be developed. However, despite the ability to induce tumor antigen-specific cytotoxic T cells, success has been very rare $(27,28)$. It is known that malignant transformation is associated with genetic alterations providing tumor cells with mechanisms for escape from immune surveillance. The data of the present studies point to a decreased susceptibility to CTL-induced lysis of metastatic melanoma cells as compared to that of their primary equivalents. These results are in agreement with the report of Thompson et al that both primary and metastatic prostate cells induced antitumor CTLs in syngeneic hosts but, unlike the primary cells, the metastatic cells were resistant to CTL lysis (29). Furthermore, the existence of a dominant factor in metastatic prostate cancer cells that is able to confer specific protection against CTL cytolysis has been suggested. One of the mechanisms for the immune escape phenotype in tumor cells is loss or reduction of class I MHC expression (30). In this regard, Takenoyama et al have reported that, although HLA class I was maintained in a primary and metastatic human lung carcinoma established from one patient, the CTL clone lysed only the primary cells (31). These results agree with our finding that, however similar the level of class I MHC molecule expression was, a significant decrease in metastatic melanoma susceptibility to killing by CTLs occurred. This suggests that, during tumor progression, metastatic cancer cells may acquire various complex mechanisms to evade cellular destruction by CTLs.

Since it has been reported that NKG2D serves as a costimulatory receptor for TCR-mediated signals in a manner similar to CD28 $(32,33)$ and that a low expression of its ligands, MICA and MICB, was responsible for the resistance of some tumor cells to lysis by effector cells (34), we asked whether an altered expression of this receptor or its ligands on metastatic cells could explain the differential susceptibility to specific cell-mediated cytotoxicity. Our data clearly indicate that primary and metastatic tumor cells express similar low levels of MICA and MICB, suggesting that the expression of NKG2D ligands by metastatic tumor cells is not involved in the down-regulated CTL reactivity induced by metastatic cells. When TNF secretion by CTLs cocultured with primary and metastatic cells was measured, the same level was observed. This suggests that a loss of recognition of the metastatic tumor is probably not involved in G1 resistance to CTL-mediated killing.

Despite the similar conjugate formation between CTL and primary or metastatic cells, we noticed a differential $\mathrm{Ca}^{2+}$ flux in the LT12 CTL clone following its interaction with T1 or G1 tumor cells. Obviously, a more pronounced increase in calcium influx was observed in the presence of the primary cells. As calcium influx has been shown to play an important 
role in the induction of CTL activity (25), it is tempting to speculate that the differential susceptibility of primary and metastatic melanoma to killing by CTL can be explained, at least in part, by a more efficient functional CTL interaction with the primary versus the metastatic clone. Such interaction would be important to trigger the lytic potential of CTLs. We observed a stronger expression of perforin and granzyme B mRNA in the CTLs when cocultered in the presence of the primary cells as compared to metastatic tumors, suggesting that primary cells have a more pronounced stimulatory potential to trigger cytotoxic molecule transcription in effector cells. Nevertheless, monitoring the presence or absence of perforin and granzyme gives no indication of the ability of $\mathrm{T}$ cells to degranulate. So, we examined the degranulation of the CTL clone (LT12) at different times of coculture with T1 and G1. We demonstrated that CTLs displayed the same degranulation capacity in the presence of primary and metastatic cells. This suggests the absence of an inhibitory factor of degranulation in CTLs in the presence of metastatics cells which was considered one of the mechanisms of immune escape (35).

Overall, our data indicate that the differential response to CTL-mediated cytolysis may be related to a difference in cytotoxic molecule transcription following CTL recognition of primary and metastatic tumor cells. Immune response induced by immunotherapy may eliminate a majority of primary cancer cells but a proportion of metastatic cancer cells could remain resistant to immune attack. Therefore, to optimise immunotherapy strategies, correction of immune activating signals, eradication of inhibitory factors and evasion from newly developed immunoresistant tumor phenotypes need to be simultaneously considered.

\section{Acknowledgments}

We would like to thank Yann Lecluse for flow cytometry analysis. This work was supported in part by grants from ARC (Association pour la Recherche sur le Cancer No. 3272) and Ligue contre le Cancer (Comité de Seine Saint-Denis).

\section{References}

1. Jemal A, Clegg LX, Ward E, et al: Annual report to the nation on the status of cancer, 1975-2001, with a special feature regarding survival. Cancer 101: 3-27, 2004.

2. Rosenberg SA, Yang JC and Restifo NP: Moving beyond current vaccines. Nat Med Cancer Immunother 10: 909-915, 2004.

3. Carr KM, Bittner M and Trent JM: Gene-expression profiling in human cutaneous melanoma. Oncogene 22: 3076-3080, 2003.

4. Wang E, Miller LD, Ohnmacht GA, et al: Prospective molecular profiling of melanoma metastases suggests classifiers of immune responsiveness. Cancer Res 62: 3581-3586, 2002.

5. Hunter CA and Reiner SL: Cytokines and T cells in host defense. Curr Opin Immunol 12: 413-418, 2000.

6. Trapani JA and Smyth MJ: Functional significance of the perforin/granzyme cell death pathway. Nat Rev Immunol 2: 735-747, 2002.

7. Lieberman J: The ABCs of granule-mediated cytotoxicity: new weapons in the arsenal. Nat Rev Immunol 3: 361-370, 2003.

8. Ashkenazi A: Targeting death and decoy receptors of the tumornecrosis factor superfamily. Nat Rev Cancer 2: 420-430, 2002.

9. Chouaib S, Asselin-Paturel C, Mami-Chouaib F, Caignard A and Blay JY: The host-tumor immune conflict: from immunosuppression to resistance and destruction. Immunol Today 18 : 493-497, 1997.
10. Glinsky GV: Apoptosis in metastatic cancer cells. Crit Rev Oncol Hematol 25: 175-186, 1997.

11. Glinsky GV and Glinsky VV: Apoptosis and metastasis: a superior resistance of metastatic cancer cells to programmed cell death. Cancer Lett 101: 43-51, 1996.

12. Glinsky GV, Glinsky VV, Ivanova AB and Hueser CJ: Apoptosis and metastasis: increased apoptosis resistance of metastatic cancer cells is associated with the profound deficiency of apoptosis execution mechanisms. Cancer Lett 115: 185-193, 1997.

13. Wong CW, Lee A, Shientag L, et al: Apoptosis: an early event in metastatic inefficiency. Cancer Res 61: 333-338, 2001.

14. Takasu M, Tada Y, Wang JO, Tagawa M and Takenaga K: Resistance to apoptosis induced by microenvironmental stresses is correlated with metastatic potential in Lewis lung carcinoma. Clin Exp Metastasis 17: 409-416, 1999.

15. Hu YP, Haq B, Carraway KL, Savaraj N and Lampidis TJ: Multidrug resistance correlates with overexpression of Muc4 but inversely with P-glycoprotein and multidrug resistance related protein in transfected human melanoma cells. Biochem Pharmacol 65: 1419-1425, 2003.

16. Douma S, Van Laar T, Zevenhoven J, Meuwissen R, van Garderen E and Peeper DS: Suppression of anoikis and induction of metastasis by the neurotrophic receptor TrkB. Nature 430: 1034-1039, 2004.

17. Dufour E, Carcelain G, Gaudin C, Flament C, Avril MF and Faure F: Diversity of the cytotoxic melanoma-specific immune response: some CTL clones recognize autologous fresh tumor cells and not tumor cell lines. J Immunol 158: 3787-3795, 1997.

18. Pross HF, Baines MG, Rubin P, Shragge P and Patterson MS: Spontaneous human lymphocyte-mediated cytotoxicity against tumor target cells. IX. The quantitation of natural killer cell activity. J Clin Immunol 1: 51-63, 1981.

19. Dorothee G, Echchakir H, Le Maux Chansac B, et al: Functional and molecular characterization of a KIR3DL2/ p140 expressing tumor-specific cytotoxic T lymphocyte clone infiltrating a human lung carcinoma. Oncogene 22: 7192-7198, 2003.

20. Echchakir H, Dorothee G, Vergnon I, Menez J, Chouaib S and Mami-Chouaib F: Cytotoxic T lymphocytes directed against a tumor-specific mutated antigen display similar HLA tetramer binding but distinct functional avidity and tissuedistribution. Proc Natl Acad Sci USA 99: 9358-9363, 2002.

21. Guerra N, Michel F, Gati A, et al: Engagement of the inhibitory receptor CD158a interrupts TCR signaling preventive dynamic membrane reorganization in CTL/tumor cell interaction. Blood 100: 2874-2881, 2002.

22. Betts MR, Brenchley JM, Price DA, De Rosa SC, Douek DC, Roederer M and Koup RA: Sensitive and viable identification of antigen-specific $\mathrm{CD}^{+} \mathrm{T}$ cells by a flow cytometric assay for degranulation. J Immunol Methods 281: 65-78, 2003.

23. Ferrone S and Marincola FM: Loss of HLA class I antigens by melanoma cells: molecular mechanisms, functional significance and clinical relevance. Immunol Today 16: 487-494, 1995.

24. Van Duinen SG, Ruiter DJ, Broecker EB, van der Velde EA, Sorg C, Welvaart K and Ferrone S: Level of HLA antigens in locoregional metastases and clinical course of the disease in patients with melanoma. Cancer Res 48: 1019-1025, 1988.

25. Xavier R, Brennan T, Li Q, McCormack C and Seed B: Membrane compartmentation is required for efficient $\mathrm{T}$ cell activation. Immunity 8: 723-732, 1998.

26. Neidhardt-Berard EM and Negrier S: [Disseminated melanoma treatment]. Rev Prat 54: 1210-1214, 2004.

27. Houghton AN, Gold JS and Blachere NE: Immunity against cancer: lessons learned from melanoma. Curr Opin Immunol 13: 134-140, 2001.

28. Nielsen MB and Marincola FM: Melanoma vaccines. the paradox of $\mathrm{T}$ cell activation without clinical response. Cancer Chemother Pharmacol 46: S62-S66, 2000.

29. Lee HM, Timme TL and Thompson TC: Resistance to lysis by cytotoxic T cells: a dominant effect in metastatic mouse prostate cancer cells. Cancer Res 60: 1927-1933, 2000.

30. Bodmer WF, Browning MJ, Krausa P, Rowan A, Bicknell DC and Bodmer JG: Tumor escape from immune response by variation in HLA expression and other mechanisms. Ann NY Acad Sci 690: 42-49, 1993.

31. So T, Takenoyama M, Ichiki Y, et al: A different pattern of cytotoxic $\mathrm{T}$ lymphocyte recognition against primary and metastatic tumor cells in a patient with non-small cell lung carcinoma. Cancer 103: 200-208, 2005. 
32. Groh V, Rhinehart R, Randolph-Habecker J, Topp MS, Riddell SR and Spies T: Costimulation of CD8alphabeta T cells by NKG2D via engagement by MIC induced on virus-infected cells. Nat Immunol 2: 255-260, 2001.

33. Maasho K, Opoku-Anane J, Marusina AI, Coligan J and Borrego F: NKG2D is a costimulatory receptor for human naive CD8 ${ }^{+}$T cells. J Immunol 174: 4480-4484, 2005.
34. Romanski A, Bug G, Becker S, et al: Mechanisms of resistance to natural killer cell-mediated cytotoxicity in acute lymphoblastic leukemia. Exp Hematol 33: 344-352, 2005.

35. Bladergroen BA, Meijer CJ, ten Berge RL, et al: Expression of the granzyme B inhibitor, protease inhibitor 9 , by tumor cells in patients with non-Hodgkin and Hodgkin lymphoma: a novel protective mechanism for tumor cells to circumvent the immune system? Blood 99: 232-237, 2002. 